\title{
Reproductive character displacement is not the only possible outcome of reinforcement
}

\author{
A. R. LEMMON, ${ }^{*}$ C. SMADJA $† \&$ M. KIRKPATRICK* \\ * Section of Integrative Biology, University of Texas, Austin, TX, USA \\ †Institut de Science de l'Evolution, Université Montpellier, Montpellier, France
}

Keywords:

hybridization;

hybrid zone;

cline;

sexual selection;

speciation.

\begin{abstract}
We study the form of the clines in a female mating preference and male display trait using simulations of a hybrid zone. Allopatric populations of two species are connected by demes in a stepping stone arrangement. Results show that reproductive character displacement (a pattern of increased prezygotic isolation in sympatry compared with allopatry) may or may not result when there is reinforcement (defined here as the strengthening of prezygotic isolation as a result of selection against hybrids, relative to the amount of prezygotic isolation present when hybrids are not selected against). Further, reproductive character displacement of the preference may or may not occur when it occurs in the male display. We conclude that the absence of reproductive character displacement is not evidence against the operation of reinforcement.
\end{abstract}

\section{Introduction}

Secondary contact between partially reproductively isolated populations may have several possible outcomes: extinction of one of the two populations, stable coexistence with hybridization, fusion of the two populations, or an increase in premating divergence and formation of distinct species (Barton \& Hewitt, 1981; Liou \& Price, 1994). In 1940, Dobzhansky postulated that if two divergent populations produce hybrids of low fitness where they come into contact, natural selection will enhance premating isolation (Dobzhansky, 1940; Howard, 1993). This process was termed reinforcement by Blair (1955).

Testing the plausibility of reinforcement has been a challenge for theoretical researchers. Reinforcement was long considered to be controversial because it was thought that very strong selection was required to compensate for the negative effect of recombination and gene flow (Paterson, 1978; Spencer et al., 1986). More recently, however, an increasing number of new models have moved the hypothesis of reinforcement into the

Correspondence: Alan R. Lemmon, Section of Integrative Biology, The University of Texas at Austin, 1 University Station no. C0930, Austin, TX 78712, USA.

Tel.: 512-471-3760; fax: 512-471-3878; e-mail: lemmon@evotutor.org foreground of speciation research by demonstrating the plausibility of this process under more realistic conditions (for a review, see Turelli et al., 2001; Kirkpatrick \& Ravigné, 2002).

Demonstrating reinforcement in nature is not trivial. As a result, empirical researchers have focused on one pattern that may result from reinforcement: reproductive character displacement (RCD), which is a pattern of greater divergence of an isolating trait in areas of sympatry between closely related taxa than in areas of allopatry (Brown \& Wilson, 1956; Howard, 1993). Howard emphasizes the distinction between reinforcement as a process and RCD as one potential pattern that can result from this process. With the difficulty of demonstrating reinforcement in mind, Howard focuses on RCD while omitting discussion of other patterns that may result from reinforcement.

The search for reinforcement in nature has become confused for five reasons. The first source of confusion stems from arguments regarding whether the term reinforcement should be used in cases where postzygotic isolation is already complete. Butlin $(1987 a, b)$, for example, has argued that the evolution of prezygotic isolation in this case should be termed reproductive character displacement. His reason is that speciation by reinforcement cannot occur because complete postzygotic isolation implies that the two entities are already 
distinct species. As Howard (1993) points out, however, the process of reinforcement itself may still occur since selection against hybridization can still lead to the evolution of increased prezygotic isolation. We agree with Howard's argument that the processes of speciation and reinforcement can operate independently and therefore use of the term reinforcement is appropriate when postzygotic isolation is already complete. The second reason for confusion is the failure to recognize that RCD can result from processes other than reinforcement, for example when there is interference between the mate recognition signals of taxa that do not hybridize. Howard (1993) addresses this issue and outlines the evidence needed to demonstrate that a pattern of RCD observed has resulted from the process of reinforcement.

The third reason for confusion is the failure to recognize that reinforcement can lead to patterns other than RCD. This confusion typically manifests itself in the false idea that in order for reinforcement to occur, prezygotic isolation in sympatry must be strengthened beyond the degree of prezygotic isolation seen in allopatry (Noor, 1999). We argue here that the evolutionary process by which prezygotic isolating barriers are strengthened by selection against hybridization is the same regardless of whether RCD is the resulting pattern. We, therefore, define reinforcement broadly as the strengthening of prezygotic isolation as a result of selection against hybrids, relative to the amount of prezygotic isolation present when hybrids are not selected against. By our definition, therefore, reinforcement can occur even when prezygotic isolation in sympatry is not strengthened beyond the degree of prezygotic isolation seen in allopatry. The reason is that selection against hybrids can result in fewer heterospecific matings (and thus increased prezygotic isolation) regardless of whether or not RCD is present.

A fourth reason for confusion is the failure to recognize that patterns of divergence in the female mating preference may differ from those in the male display trait. Finally, some workers have failed to recognize the role that sexual selection can play in generating selection against hybrids. If hybrids have decreased mating opportunities or decreased fertility, then reinforcement may occur even in the absence of viability selection against hybrids (Coyne \& Orr, 1989; Liou \& Price, 1994; Kirkpatrick \& Servedio, 1999; Noor, 1999).

Here we present a simple model of a hybrid zone with the aim of clarifying two of these causes of confusion. We use simulations to make the following points: that reinforcement can lead to patterns other than RCD, and that the male display trait and female preference do not always evolve the same pattern of divergence. We wish to caution empiricists not to draw conclusions about the influence of selection against hybrids on prezygotic isolation based solely on presence or absence of RCD. We conclude with a discussion of some possible disparities among researchers in the interpretation of the definition of reinforcement and some problems that may arise as a result of this disparity.

\section{The model}

We begin by assuming a simple genetic system of three diploid loci with free recombination. The three loci are a male trait locus $(\mathrm{T})$, a female preference locus $(\mathrm{P})$, and a hybrid incompatibility locus (I). We denote the two alleles at the male trait locus as $\mathrm{T}_{0}$ and $\mathrm{T}_{1}$ (with frequencies $t_{0}$ and $t_{1}$ ), those at the preference locus as $\mathrm{P}_{0}$ and $\mathrm{P}_{1}$ (with frequencies $p_{0}$ and $p_{1}$ ), and those at the hybrid incompatibility locus as $\mathrm{I}_{0}$ and $\mathrm{I}_{1}$ (with frequencies $i_{0}$ and $i_{1}$ ). Allopatric divergence at the trait locus, denoted $\Delta T$, is defined to be the difference between $t_{0}$ in the two allopatric populations. Allopatric divergence at the preference locus, $\Delta P$, and incompatibility locus, $\Delta I$, are defined in a similar fashion.

The hybrid zone is represented by 10 populations arranged in a stepping stone model, with the left and right-most populations being identified as allopatric, and all others as sympatric. The order of events in each generation is migration, natural selection, and sexual selection (mating). Migration is one-way from allopatry to sympatry but two-way in sympatry. The rate of migration between adjacent populations is denoted $m$. The effective size of each population is infinite.

We assume that the trait locus is under both natural and sexual selection. A linear environmental gradient causes viability selection that favours allele $T_{1}$ in the left allopatric population and $\mathrm{T}_{0}$ in the right allopatric population. The relative viabilities of the genotypes $\mathrm{T}_{0} \mathrm{~T}_{0}, \mathrm{~T}_{0} \mathrm{~T}_{1}$, and $\mathrm{T}_{1} \mathrm{~T}_{1}$ in the left allopatric population are $1-s_{\mathrm{T}}, 1-s_{\mathrm{T}} / 2$, and 1 , respectively, while in the right allopatric population the viabilities of the $T_{0} T_{0}$ and $T_{1} T_{1}$ males are reversed. Viabilities of the three genotypes change in a linear fashion as one moves across the hybrid zone.

Females choose their mates based on their genotype at the preference locus $\mathrm{P}$, with the $\mathrm{P}_{0}$ allele conferring a preference for males that carry the $T_{0}$ allele and the $P_{1}$ allele a preference for the $T_{1}$ allele. The frequency of matings between different male and female genotypes is proportional to the product of the frequencies of those genotypes and the preference that the female has for the male. Table 1 shows these preferences.

We assume conditions that produce a stable polymorphism in the preference and trait loci in the allopatric populations, thus permitting the preference and trait to evolve to more extreme values in sympatry (i.e. the pattern of RCD). Over-dominant natural selection acts on the female preference locus with viabilities in the left allopatric population of $1 / 2,1$, and $1-s_{\mathrm{P}}$, for the genotypes $\mathrm{P}_{0} \mathrm{P}_{0}, \mathrm{P}_{0} \mathrm{P}_{1}$, and $\mathrm{P}_{1} \mathrm{P}_{1}$, respectively. Conversely, in the right allopatric population the viabilities of the $\mathrm{P}_{0} \mathrm{P}_{0}$ and $\mathrm{P}_{1} \mathrm{P}_{1}$ females are reversed. We chose the value for $s_{\mathrm{P}}$ in order to give the desired value of $\Delta P$. Natural selection does not act on the female preference locus in sympatry. 
Table 1 The frequency of matings between different male and female genotypes.

\begin{tabular}{llll}
\hline & \multicolumn{3}{l}{ Male genotype } \\
\cline { 2 - 4 } & $\mathrm{T}_{0} \mathrm{~T}_{0}$ & $\mathrm{~T}_{0} \mathrm{~T}_{1}$ & $\mathrm{~T}_{1} \mathrm{~T}_{1}$ \\
\hline Female genotype & $(1+\alpha)^{2}$ & $(1+\alpha)$ & 1 \\
$\mathrm{P}_{0} \mathrm{P}_{0}$ & 1 & 1 & 1 \\
$\mathrm{P}_{0} \mathrm{P}_{1}$ & 1 & $(1+\alpha)$ & $(1+\alpha)^{2}$ \\
$\mathrm{P}_{1} \mathrm{P}_{1}$ & 1 & & \\
\hline
\end{tabular}

Selection against hybrids provides the force that may lead to reinforcement. Here we assume that viability selection against hybrids is disruptive, acting against heterozygotes at the incompatibility locus. The relative viabilities of the genotypes $\mathrm{I}_{0} \mathrm{I}_{0}, \mathrm{I}_{0} \mathrm{I}_{1}$, and $\mathrm{I}_{1} \mathrm{I}_{1}$ are respectively $1,1-s_{\mathrm{I}}$, and 1 in all populations, where $s_{\mathrm{I}}$ is the strength of viability selection against hybrids. The left allopatric population is fixed for allele $\mathrm{I}_{0}$, and the right for allele $I_{1}$.

Using simulations, we track genotype frequencies through time. At the beginning of each simulation, allopatric populations are brought to equilibrium. We then allow secondary contact by allowing migration into the sympatric populations. We ran the simulations until all populations reached equilibrium.

\section{Quantifying cline shape}

Figure 1 gives the interpretation of our measures of RCD and reinforcement. To quantify the cline shape, we noted the difference between $t_{0}$ in first sympatric population on the left and the adjacent allopatric population. This quantity, which we term $b_{\mathrm{T}}$, indicates if RCD is present. A positive value of $b_{\mathrm{T}}$ means there is RCD, that is, the male trait has greater divergence in sympatry than in allopatry (a 'reversed' cline). Furthermore, we can study how the intensity of selection against hybrids affects $b_{\mathrm{T}}$ to ask if increasing selection against hybrids leads to increasing premating isolation and thus reinforcement.
We can ask similar questions about the evolution of the preference using the analogous quantity $b_{\mathrm{P}}$.

To quantify the amount of reinforcement, we compare the shape of the cline under two conditions: when selection acts against hybrids $\left(s_{\mathrm{I}}>0\right)$ and when selection does not act against hybrids $\left(s_{\mathrm{I}}=0\right)$. This quantity, which we define as $\delta=b_{s}>0-b_{s}=0$ (the slope observed when selection is acting against hybrids minus the slope observed when selection is not acting against hybrids), tells us the effect of selection against hybrids on the shape of the cline. When prezygotic isolation is strengthened by selection against hybrids (reinforcement), we expect $\delta$ to be positive for either the male trait or female preference.

\section{Results}

A first basic observation from the simulations is that reinforcement does not always produce RCD. This result can be seen in Fig. 2, which presents the evolution of a cline through time under two different conditions. With no selection acting against hybrids (generation 0 ), a simple cline forms. After selection against hybrids is introduced, reinforcement occurs (generations 50 and 100) and the cline steepens. Under moderate selection against hybrids $\left(s_{\mathrm{I}}=0.65\right)$, the resulting cline at equilibrium is monotonic. Under more intense selection against hybrids $\left(s_{\mathrm{I}}=0.90\right)$, the resulting cline is reversed. We say that reinforcement has occurred in both cases because selection against hybrids resulted in increased prezygotic isolation, relative to the amount of prezygotic isolation present when hybrids are not selected against (compare equilibrium and generation $0)$. RCD, conversely, is only evident in one of the two cases.

Figure 3 and Table 2 also show cases in which there is reinforcement (i.e. increasing intensity of selection against hybrids leads to increased divergence of the preference and/or male trait in sympatry) but not RCD (i.e. greater divergence in sympatry than allopatry, indicated by positive values of $b_{\mathrm{T}}$ and/or $b_{\mathrm{P}}$ ). Under a
Fig. 1 Measures of reproductive character displacement and reinforcement. The left panel shows the interpretations for $b$, our measure of cline shape, and the right panel shows the interpretations for $\delta$, our measure of reinforcement. A dashed line (- -) indicates that no selection is acting against hybrids, whereas a solid line (-) indicates that hybrids have reduced fitness.

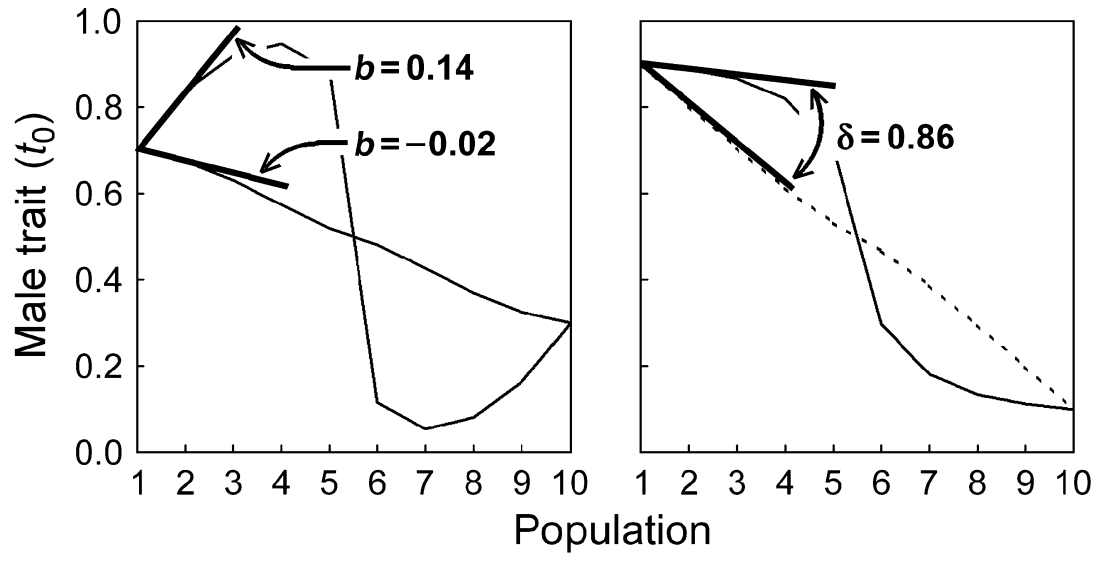


(a)
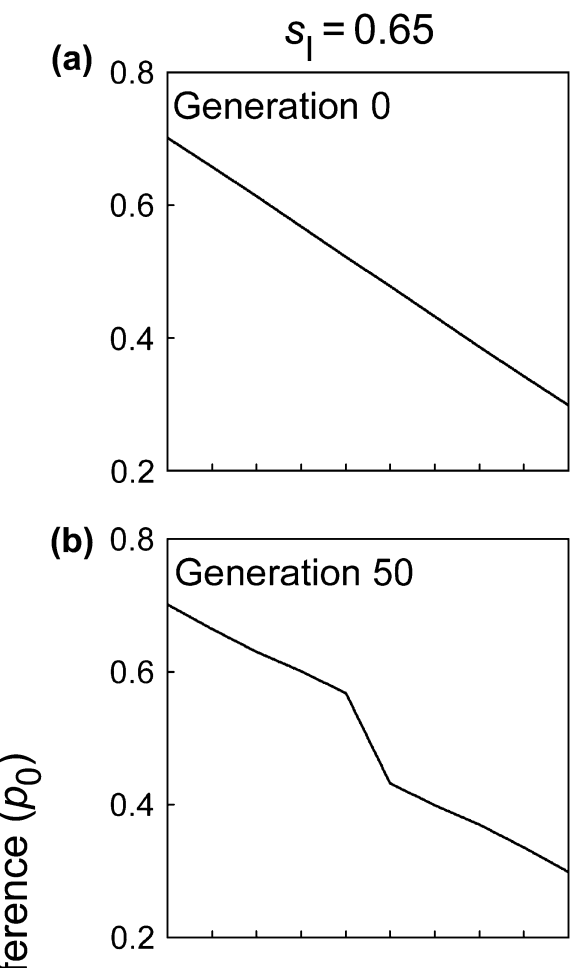

(c)

$\frac{0}{\frac{1}{0}}$

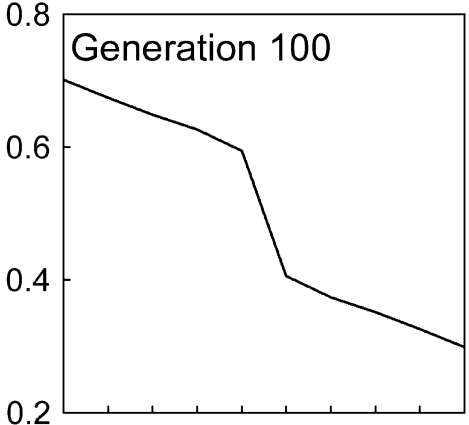

(d)

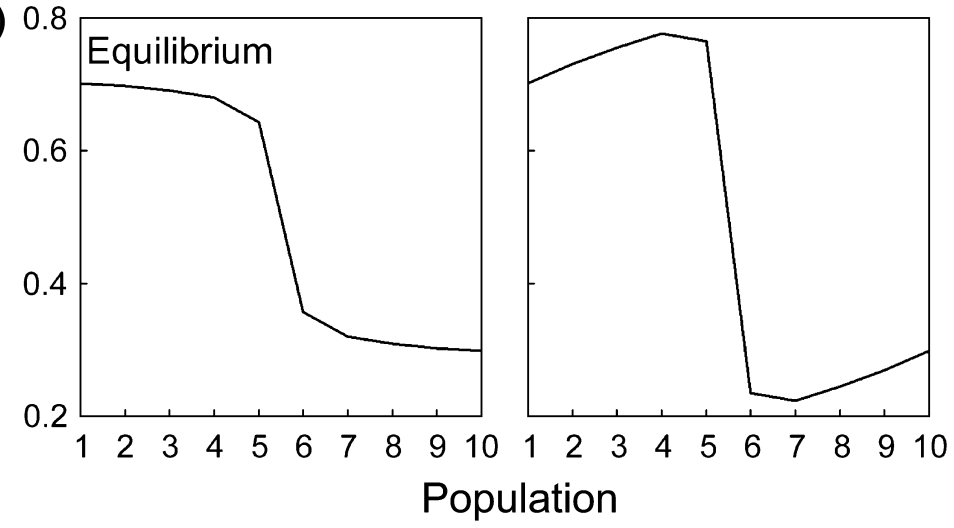

Fig. 2 The evolution of a cline in response to selection against hybridization. (a) A simple cline in mating preferences forms when there is no selection against hybrids. (b \& c) Selection against hybrids results in reinforcement of mating preferences in sympatry. (d) The resulting shape of the cline at equilibrium depends upon a number of conditions, including the strength of selection against hybrids $\left(s_{\mathrm{I}}\right)$. Under some conditions $\left(s_{\mathrm{I}}=0.90\right)$, the resulting cline shows the pattern of reproductive character displacement whereas in others $\left(s_{\mathrm{I}}=0.65\right)$, the resulting cline is monotonic. The process leading to the strengthening of prezygotic isolation in sympatry is the same in both cases. Parameter values are $\alpha=0.4$, $m=0.05, \Delta T=0.4$, and $\Delta P=0.4$. broad range of conditions, viability selection against hybrids $\left(s_{\mathrm{I}}\right)$ leads to reinforcement of both the preference and male trait, indicated by positive values of $\delta_{\mathrm{T}}$ and $\delta_{\mathrm{P}}$.
A second basic observation is that the clines in the preference and male trait can be qualitatively different in shape. Under some conditions, both the preference 

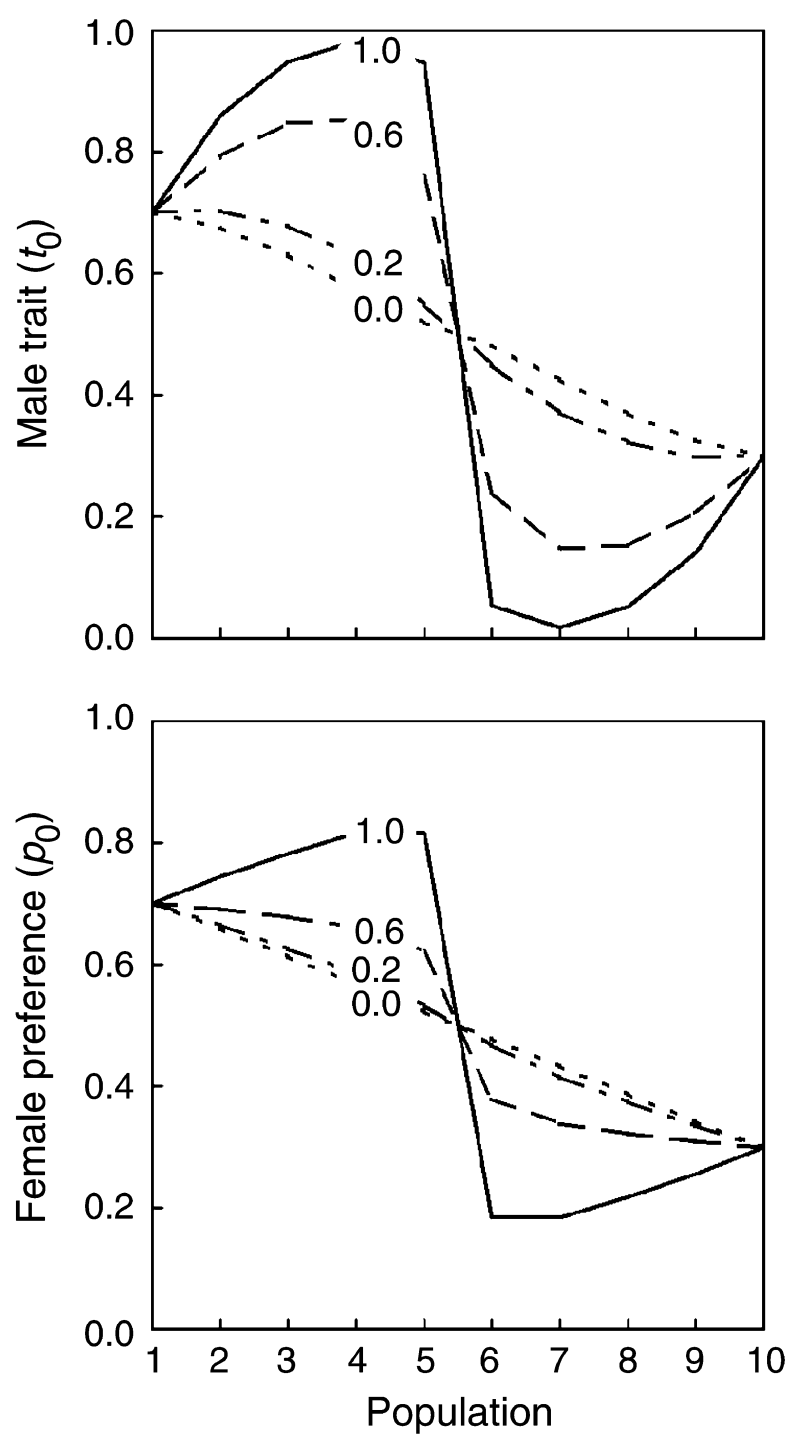

Fig. 3 Patterns of divergence in the male trait (above) and female preference (below). The four clines in each panel correspond to different intensities of viability selection against hybrids (with the value for $s_{\mathrm{I}}$ shown on each curve). Parameter values are $\alpha=0.4$, $m=0.05, \Delta T=0.4$, and $\Delta P=0.4$.

and male trait show RCD, while under others only the male trait does. Examples are shown in Fig. 3. Although we typically observed monotonic and reversed clines, we also observed a third type of cline, in which the pattern of divergence in sympatry was the reverse of that in allopatry. Figure 4 shows examples of different patterns that can be produced by reinforcement. We also observed that the male trait consistently diverges more than the preference in our model. That may not be a general pattern, however, as the quantitative outcome may depend on behavioural and genetic details.
The simulations also suggest the roles that different parameters may have in promoting RCD and reinforcement. Table 2 shows that the degree of RCD in the male trait and female preference is enhanced by increased female mating preferences (larger $\alpha$ ) and by larger differences between the average female mating preferences of the allopatric populations (larger $\Delta P$ ). Increased divergence of the trait in allopatry $(\Delta T)$, however, generally leads to a decrease in the amount of RCD observed for the male trait and female preference (smaller $b_{\mathrm{T}}$ and $b_{\mathrm{P}}$ ). As might be anticipated, increased migration $(m)$ leads to decreased reinforcement of both the male trait and preference (smaller $\delta_{\mathrm{T}}$ and $\delta_{\mathrm{P}}$ ). Our simulations do not allow us to say how these conclusions might generalize to other assumptions regarding the genetics, geography, behaviour, etc.

\section{Discussion}

The model presented here demonstrates two simple points. First, the absence of RCD does not imply the absence of reinforcement. This result makes the point that RCD is not the unique signature of reinforcement. Second, the preference and male trait can have qualitatively different clines. This result underlines the importance of studying the patterns of divergence in both the trait and the preference. While the quantitative results we find depend on the detailed assumptions made in our model, we expect that these two qualitative conclusions may hold under a broad range of conditions.

We have focused here on the concept of reinforcement originated by Dobzhansky (1940), defined by Blair (1955), and further clarified by Howard (1993). This definition describes the process that selection against hybrids may lead to: the enhancement of prezygotic isolation. We have shown that prezygotic isolation can be enhanced by selection against hybrids without resulting in RCD, defined as the pattern in which there is more divergence in sympatry than allopatry (Brown 8 Wilson, 1956).

There may be a tendency, because of empirical convenience, to equate reinforcement with RCD. This has led to a conception of reinforcement as an increase in prezygotic isolation in sympatry relative to allopatry, because of selection against hybrids. Use of this definition leads researchers to view RCD as a necessary outcome of reinforcement. This definition also downplays the importance of the evolution of prezygotic isolation in the stages where RCD is not present. During these stages the frequency of heterospecific matings is reduced and fewer hybrid zygotes are formed just as they are when RCD is present. Our simulations show that RCD is just one possible outcome that can result from selection against hybrids. We see no difference between the process that steepens a monotonic cline and the process that transforms a monotonic cline into a reversed cline. In both cases the propensity to mate with conspecifics 


\begin{tabular}{lllllrlll}
\hline$\alpha$ & $m$ & $\Delta T$ & $\Delta P$ & $s_{\mathrm{I}}$ & \multicolumn{1}{l}{$b_{\mathrm{T}}$} & \multicolumn{1}{l}{$b_{\mathrm{P}}$} & \multicolumn{1}{l}{$\delta_{\mathrm{T}}$} & \multicolumn{1}{l}{$\delta_{\mathrm{P}}$} \\
\hline 0.4 & 0.050 & 0.4 & 0.4 & 0.4 & 0.0364 & -0.0279 & 0.0611 & 0.0153 \\
0.2 & - & - & - & - & -0.1437 & -0.0420 & 0.0183 & 0.0081 \\
0.8 & - & - & - & - & 0.1643 & 0.0012 & 0.0589 & 0.0263 \\
1.0 & - & - & - & - & 0.1869 & 0.0128 & 0.0531 & 0.0303 \\
- & 0.025 & - & - & - & 0.0939 & -0.0217 & 0.1013 & 0.0202 \\
- & 0.050 & - & - & - & 0.0364 & -0.0279 & 0.0611 & 0.0153 \\
- & 0.075 & - & - & - & 0.0153 & -0.0295 & 0.0464 & 0.0142 \\
- & 0.100 & - & - & - & 0.0050 & -0.0299 & 0.0393 & 0.0141 \\
- & - & 0.2 & - & - & 0.0748 & -0.0269 & 0.0614 & 0.0152 \\
- & - & 0.4 & - & - & 0.0364 & -0.0279 & 0.0611 & 0.0153 \\
- & - & 0.6 & - & - & -0.0011 & -0.0289 & 0.0612 & 0.0154 \\
- & - & 0.8 & - & - & -0.0364 & -0.0298 & 0.0616 & 0.0156 \\
- & - & - & 0.2 & - & -0.1942 & -0.0335 & 0.0002 & 0.0017 \\
- & - & - & 0.4 & - & 0.0364 & -0.0279 & 0.0611 & 0.0153 \\
- & - & - & 0.6 & - & 0.1368 & -0.0384 & 0.0454 & 0.0191 \\
- & - & - & - & 0.0 & -0.0248 & -0.0432 & 0.0000 & 0.0000 \\
- & - & - & - & 0.2 & 0.0021 & -0.0363 & 0.0269 & 0.0069 \\
- & - & - & - & 0.6 & 0.0940 & -0.0098 & 0.1188 & 0.0334 \\
- & - & - & - & 0.8 & 0.1366 & 0.0157 & 0.1614 & 0.0589 \\
- & - & - & - & 1.0 & 0.1594 & 0.0435 & 0.1842 & 0.0867 \\
\hline
\end{tabular}

Subscripts $\mathrm{T}$ and $\mathrm{P}$ correspond to the male trait and female preference loci, respectively. Parameters include the strength of female preference $(\alpha)$, rate of migration $(m)$, amount of allopatric divergence in the male trait $(\Delta T)$ and female preference $(\Delta P)$, and strength of viability selection against hybrids $\left(s_{\mathrm{I}}\right)$.

Each row presents the parameter values assumed and the outcome of one simulation, where a dash (-) indicates a parameter value that is equal to the value in the top row.

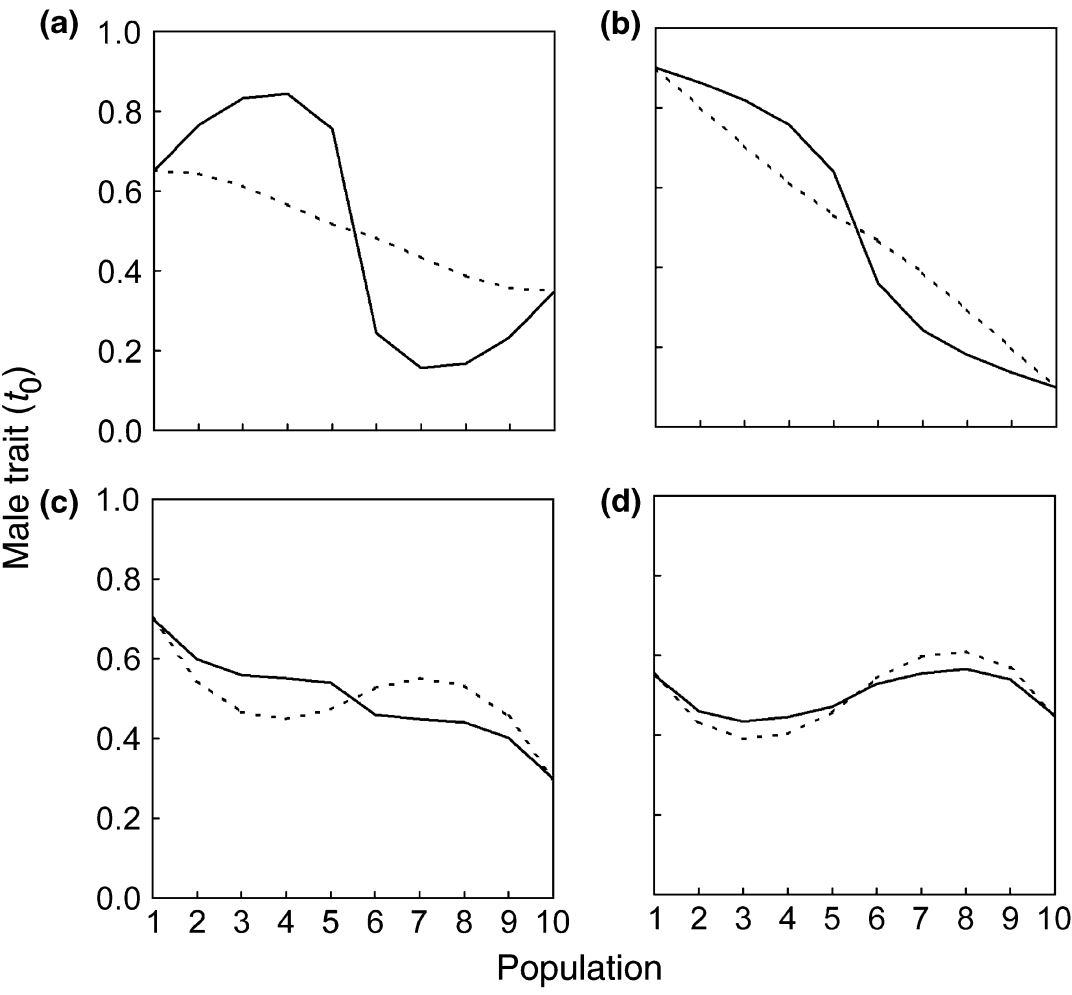

Table 2 Cline shape $(b)$ and the amount of reinforcement $(\delta)$ under different evolutionary assumptions.
Fig. 4 Equilibrium cline shapes resulting from reinforcement. A dashed line (- -) indicates that no selection is acting against hybrids, whereas a solid line (-) indicates that hybrids have reduced fitness. Reinforcement can result in (a) a reversed cline, (b) a monotonic cline, or (c \& d) other types of clines. Note that although reinforcement occurred in all four of these cases, reproductive character displacement resulted in only one case (a). Parameter values are (a) $\alpha=0.4, m=0.05, \Delta T=0.3, \Delta P=0.4$, and $s_{\mathrm{I}}=0.6$, (b) $\alpha=0.4, m=0.05, \Delta T=0.8$, $\Delta P=0.4$, and $s_{\mathrm{I}}=0.4$, (c) $\alpha=0.4$, $m=0.05, \Delta T=0.4, \Delta P=0.3$, and $s_{\mathrm{I}}=0.6$, and (d) $\alpha=0.2, m=0.05, \Delta T=0.1$, $\Delta P=0.4$, and $s_{\mathrm{I}}=0.6$. 
evolves by indirect selection generated by direct selection on less fit hybrids.

As Howard (1993) and Noor (1999) point out, RCD may result from evolutionary processes other than reinforcement. It is therefore critical to distinguish between the evolutionary process that enhances isolation, which may or may not be reinforcement, and the pattern of the outcome, which may or may not be RCD. This is not to say that the study of RCD in nature is not important; we may indeed learn volumes about speciation by concentrating on geographical regions of enhanced prezygotic isolation. The point is just that we cannot draw conclusions about whether reinforcement has occurred based solely on the presence or absence of RCD.

How then can we determine if reinforcement has occurred in nature? Ideally, we might want to compare the hybrid zone of interest with another zone that is identical but that has no selection against hybrids. One could, for example, compare the proportion of heterospecific matings in sympatry under the two cases, expecting to see fewer where there is selection against hybrids. Alternatively, we could compare the slope of the cline under the two cases. We would expect greater divergence (i.e. larger values of $b_{\mathrm{T}}$ and/or $b_{\mathrm{p}}$ ) when selection is acting against hybrids than when selection is not. While these approaches are practical for simulation studies, they clearly are not feasible in nature.

Perhaps asking whether reinforcement has occurred is not the most informative question. Existing analytical models of reinforcement show that prezygotic isolation is strengthened by selection acting against hybrids under very general conditions (Kirkpatrick \& Servedio, 1999; Kirkpatrick, 2000, 2001), and our findings are consistent with that conclusion. These results suggest we can expect there will be some reinforcement whenever there is some assortative mating and some selection against hybrids. If our aim is to understand the role of postzygotic isolation in the formation and maintenance of species, then perhaps we should not be asking whether or not reinforcement is occurring, but instead be trying to understand what effect reinforcement has on the patterns of divergence we see in nature.

\section{Acknowledgments}

This research was supported by NSF grant DEB-9973221 to M.K. and an NSF Graduate Research Fellowship to
A.R.L. C.S. was supported by CNRS (UMR 5554) through Guila Ganem. N ${ }^{\circ}$ ISEM 2003-061. We thank Guila Ganem for many helpful discussions.

\section{References}

Barton, N.H. \& Hewitt, G.M. 1981. Hybrid zones and speciation. In: Evolution and Speciation/Essays in Honor of M.J.D. White (W. R. Atchley \& D. S. Woodruff, eds), pp. 109-145. Cambridge University Press, Cambridge.

Blair, W.F. 1955. Mating call and stage of speciation in the Microhyla olivacea M. carolinensis complex. Evolution 9: 469-480.

Brown, W.L. Jr. \& Wilson, E.O. 1956. Character displacement. Syst. Zool. 5: 49-64.

Butlin, R.K. 1987a. Speciation by reinforcement. Trends Ecol. Evol. 2: 8-13.

Butlin, R.K. 1987b. Species, speciation, and reinforcement. Am. Nat. 130: 461-464.

Coyne, J.A. \& Orr, H.A. 1989. Patterns of speciation in Drosophila. Evolution 43: 362-381.

Dobzhansky, T. 1940. Genetics and the Origin of Species. Columbia University Press, New York.

Howard, D.J. 1993. Reinforcement: origin, dynamics, and fate of an evolutionary hypothesis. In: Hybrid Zones and the Evolutionary Process (R. G. Harrison, ed.), pp. 46-69. Oxford University Press, New York.

Kirkpatrick, M. 2000. Reinforcement and divergence under assortative mating. Proc. R. Soc. Lond. B 267: 1649-1655.

Kirkpatrick, M. 2001. Reinforcement during ecological speciation. Proc. R. Soc. Lond. B 268: 1259-1263.

Kirkpatrick, M. \& Ravigné, V. 2002. Speciation by natural and sexual selection: models and experiments. Am. Nat. 159: S22S35.

Kirkpatrick, M. \& Servedio, M. 1999. The reinforcement of mating preferences on an island. Genetics 151: 1764-1772.

Liou, L.W. \& Price, T.D. 1994. Speciation by reinforcement of premating isolation. Evolution 48: 1451-1459.

Noor, M.A.F. 1999. Reinforcement and other consequences of sympatry. Heredity 83: 503-508.

Paterson, H.E.H. 1978. More evidence against speciation by reinforcement. S. Afr. J. Sci. 74: 369-371.

Spencer, H.G., McArdle, B.H. \& Lambert, B.M. 1986. A theoretical investigation of speciation by reinforcement. Am. Nat. 128: 241-262.

Turelli, M., Barton, N.H. \& Coyne, J.A. 2001. Theory and speciation. Trends Ecol. Evol. 16: 330-343.

Received 28 January 2003; revised 9 June 2003; accepted 12 August 2003 